\title{
A Mathematic Model for Dynamic Transient Response of Electromagnetic Rail on Elastic Foundation
}

\author{
Lei Shi (Corresponding author), Wen Liu \& Chunyan Zhou \\ School of Sciences, Yanshan University \\ Qinhuangdao 066004, China \\ E-mail: lovelys1840815@163.com
}

Xiangzhong Bai

School of Civil Engineering \& Mechanics, Yanshan University

Qinhuangdao 066004, China

The project support by the National Nature Science Foundation of China under Grand NO.50875230

\begin{abstract}
The electromagnetic launcher's rail can be modeled as a beam which is on elastic foundation with cantilevered support by moving load. In this paper Euler beam theory is applied to build the Mathematic model and the complete solution of the equation is derived in detail. At last, a numerical experiment, which analyzes the influences brought by the moving load velocity and the damping force on the transient response of beam, shows that the moving load's velocity has a quite obvious affect on the response of the beam.
\end{abstract}

Keywords: Electromagnetic railgun, Elastic foundation beam, Mathematic model, Damping force, Lagrange equation

\section{Introduction}

According to the characteristics of modern warfare and the new military requirements, the rise of weapons of new concept is a leap in the development of military theory and technology in recent years. The electromagnetic railgun, as an important one of new concept weapons, has adopted the electromagnetic launching method, not only broke through the velocity limitation of the projectile by the launching method of traditional chemical energy, but also greatly surpassed the rate of cannon projectile and reduced the reaction time. Hence, it has a huge enlargement in recent years. The working principle of electromagnetic launcher is very different from traditional artillery for it's using power as an energy source, such that the export speed, efficiency are greatly impacted by a number of factors. In order to fully understand the changes of performance of electromagnetic railgun, we must study deeply on it. According to the research at home and abroad in recent years, although there will be much time needed to apply the technology of electromagnetic launchers in combat, a framework of research of the overall electromagnetic launchers and local properties are built basically. To improve the electromagnetic launchers' launching speed, efficiency and to extend the service life of the barrel, the research works are focus on launching ballistic model, the key lies in the high-precision numerical calculation and analysis of the influences by a variety of factors of electromagnetic launchers on emission properties, which contribute to the design a more reasonable electromagnetic launcher(Wang, Ying\& Xiao, Feng, 1994)( Hu, Yuwei. 2007).

T.Tzeng(Jerome T. Tzeng \&Wei, Sun., 2007) used the elastic foundation beam to build mechanical model of the electromagnetic railgun and deduced the solve process of governing equation. Weng xuetao Hu'an(Weng, Xuetao \& $\mathrm{Hu}, 2008$ ) solved the governing equation's numerical solution by double Fourier transform and analyzed the response of beam which is impelled by moving vibrating mass. Yu Yanli(Yu, Yanli., 2002) adopted the Fourier transform and Laplace transform methods to study the process of calculation on elastic grade beam. The damping force to the response of beam is ignored in the above researches. In this paper, regarding the rail as a cantilever beam on the elastic foundation and considering the damping force, using propose a mechanic model which is under the effect of moving load. Moreover, making use of variable method and the Lagrange equation which considering the damping force, the complete solution of the governing equation is derived and the influences brought by the velocity of moving load and 
damping coefficient on the response of beam is analyzed.

\section{Mathematical Model}

Figure.1 shows a schematic of a electromagnetic railgun composed of power source, rail, armature and projectile. When the electric current of armature goes through the rail, it forms a strong magnetic field in the area of their encirclement. With the reaction by the magnetic field and the electric current, it emerges powerful electromagnetic force, which pushes the armature and projectile to do the accelerating motion along the rail till the projectile be launched out of the rail.

Figure .2 is the physical model of the railgun - there is a cantilever beam with one end fixed and the other end free partially subjected to even load sitting on the elastic foundation. Considering the effect of the beam by the damping force and basing on the Euler beam theory, we obtain the governing equation of elastic foundation beam by moving load which is a transient fourth —order differential equation as follows(S.Timoshenko. 1965)( Liu, Xiaoyun, Tian Runli. 2007):

$$
m \frac{\partial^{2} w}{\partial t^{2}}+E I \frac{\partial^{4} w}{\partial x^{4}}+k w+c \frac{\partial w}{\partial t}=q[1-H(x-v t)]
$$

Where $w$ is the deflection, which depend on time $t$ and position $x, m=\rho B h$ is the mass per unit length, $\rho$ is the density of rail material, $B$ and $h$ are respectively the width and thickness of the rail, $E I$ is the bending stiffness of beam, $k$ is the elastic constant, $c$ is the damping coefficient. The function $q[1-H(x-v t)]$ in(1), represents the magnetic pressure front traveling along the rail $v$ represented by a Heaviside step function $H(x-v t)$, and $q$ is a constant(Jerome T. Tzeng. 2005).

\section{Solution of the homogeneous equation}

The homogeneous equation is a fourth-order partial differential equation, in order to change it into the ordinary differential equation, we solve it by the method of variable separation.

Assume that the solution of the homogeneous equation can be expressed as follows:

$$
w(x, t)=\phi(t) \theta(x)
$$

Substituting(2)into the homogeneous equation of(1):

$$
m \frac{\partial^{2} \phi}{\partial t^{2}} \theta+E I \phi \theta^{(4)}+k \phi \theta+c \theta \frac{\partial \phi}{\partial t}=0
$$

That can be expressed as follows:

$$
-\frac{\frac{\partial^{2} \phi}{\partial t^{2}}}{c \phi}-\frac{\frac{\partial \phi}{\partial t}}{m \phi}=\frac{E I}{c m} \frac{\theta^{(4)}}{\theta}+\frac{k}{c m}
$$

from equation(4), let:

$$
-\frac{\frac{\partial^{2} \phi}{\partial t^{2}}}{c \phi}-\frac{\partial \phi}{m \phi}=\lambda^{2}
$$

And

$$
\frac{E I}{c m} \frac{\theta^{(4)}}{\theta}+\frac{k}{c m}=\lambda^{2}
$$

That is

$$
\theta^{(4)}-\beta^{4} \theta=0
$$

where

$$
\beta^{4}=\left(\lambda^{2}-\frac{k}{c m}\right) \frac{c m}{E I}
$$


solution of equation(5)can be expressed as follows:

$$
\phi(t)=A e^{\frac{-c+R}{2 m}}+B e^{\frac{-c-R}{2 m}}
$$

where:

$$
R^{2}=c^{2}-4 c m^{2} \lambda^{2}>0
$$

Based on the boundary condition of the cantilever beam,

$$
\left\{\begin{array} { c } 
{ \theta ( 0 ) = 0 } \\
{ \frac { \partial \theta ( x ) } { \partial x } | _ { x = 0 } = 0 }
\end{array} \quad \text { and } \quad \left\{\begin{array}{l}
\left.\frac{\partial^{2} \theta(x)}{\partial x^{2}}\right|_{x=L}=0 \\
\left.\frac{\partial^{3} \theta(x)}{\partial x^{3}}\right|_{x=L}=0
\end{array}\right.\right.
$$

solution of equation(7)can be expressed as follows(Zhu, Shijian, Lou, lingjun. 2006):

$$
\theta_{i}(x)=\left[\left(\cosh \beta_{i} x-\cos \beta_{i} x\right)-\frac{\cosh \beta_{i} L+\cos \beta_{i} L}{\sinh \beta_{i} L+\sin \beta_{i} L}\left(\sinh \beta_{i} x-\sin \beta_{i} x\right)\right]
$$

Accordingly:

$$
\begin{aligned}
w_{i}(x, t) & =\theta_{i}(x) \phi_{i}(t) \\
& =\left[\left(\cosh \beta_{i} x-\cos \beta_{i} x\right)-\frac{\cosh \beta_{i} L+\cos \beta_{i} L}{\sinh \beta_{i} L+\sin \beta_{i} L}\left(\sinh \beta_{i} x-\sin \beta_{i} x\right)\right] \times\left(A_{i} e^{\frac{-c+R}{2 m}}+B_{i} e^{\frac{-c-R}{2 m}}\right)
\end{aligned}
$$

In terms of the orthogonality of $\theta_{i}(x)$ ( Zhang, Xiangting, Wang, Zhipei. 2006), we obtain:

$$
\int_{0}^{L} \theta_{i} \theta_{j} d x= \begin{cases}0, & \text { at } i \neq j \\ 1, & \text { at } i=j\end{cases}
$$

Hence, deformation $w(x, t)$ of the beam can be expressed by the linear combination of $\theta_{i}(x)$.

$$
\begin{aligned}
w(x, t)= & \sum_{i} \theta_{i}(x) \phi_{i}(t) \\
= & \sum_{i}\left[\left(\cosh \beta_{i} x-\cos \beta_{i} x\right)-\frac{\cosh \beta_{i} L+\cos \beta_{i} L}{\sinh \beta_{i} L+\sin \beta_{i} L}\left(\sinh \beta_{i} x-\sin \beta_{i} x\right)\right] \\
& \times\left(A_{i} e^{\frac{-c+R}{2 m}}+B_{i} e^{\frac{-c-R}{2 m}}\right)
\end{aligned}
$$

Where constants $A_{i}, B_{i}$ are determined by the initial conditions.

\section{General Solusion}

The general solution of the homogeneous part of (1) can be derived from the above, and the general solution(1)can be obtained by the Lagrange equation which considering the damping force, where $T$ is the kinetic energy of the beam, $U$ is the total stain energy, $G$ is the dissipation function(Jin, Shangnian, Ma, Yongli. 2002).

$$
\frac{d}{d t}\left(\frac{\partial T}{\frac{\partial \phi_{i}}{\partial t}}\right)-\frac{\partial T}{\partial \phi_{i}}+\frac{\partial U}{\partial \phi_{i}}-\frac{\partial G}{\frac{\partial \phi_{i}}{\partial t}}=Q_{i}
$$

The kinetic energy of the beam $T$ can be expressed as follows(Lou, Ping \& Zeng, Qingyuan. 2003): 


$$
T=\frac{1}{2} \sum_{i} M_{i}\left(\frac{\partial \phi_{i}}{\partial t}\right)^{2}
$$

Where $M_{i}=\int_{0}^{L} m \theta_{i}^{2}(x) d x$ represents the general mass of the beam.

The total strain energy of the beam $U$ is consisted by the strain energy $U_{b}$ of the beam, and the strain energy $U_{f}$ of the foundation.

$$
\begin{gathered}
U_{b}=\frac{1}{2} \int_{0}^{L} E I\left(\frac{\partial^{2} \omega(x, t)}{\partial x^{2}}\right)^{2} d x=\frac{1}{2} \sum_{i} \sum_{j} \phi_{i} \phi_{j} \int_{0}^{L} E I \frac{\partial^{2} \theta_{i}}{\partial x^{2}} \frac{\partial^{2} \theta_{j}}{\partial x^{2}} d x=\frac{1}{2} \frac{E I}{m} \sum_{i} \beta_{i}^{4} M_{i} \phi_{i}^{2} \\
U_{f}=\frac{1}{2} \int_{0}^{L} k \omega^{2} d x=\frac{k}{2} \int_{0}^{L}\left(\sum_{i} \theta_{i} \varphi_{i}\right)^{2} d x=\frac{k}{2} \sum_{i} \sum_{j} \phi_{i} \phi_{j} \int_{0}^{L} \theta_{i} \theta_{j} d x=\frac{k}{2 m} M_{i} \sum_{i} \phi_{i}^{2}
\end{gathered}
$$

So the total strain energy $U$ is obtained as:

$$
U=U_{b}+U_{f}=\frac{1}{2} \frac{E I}{m} \sum_{i} \beta_{i}^{4} M_{i} \phi_{i}^{2}+\frac{k}{2 m} M_{i} \sum_{i} \phi_{i}^{2}=\frac{1}{2}\left[\sum_{i} M_{i} \phi_{i}^{2}\left(\frac{E I}{m} \beta_{i}^{4}+\frac{k}{m}\right)\right]=\frac{1}{2}\left[\sum_{i} c M_{i} \phi_{i}^{2} \lambda_{i}^{2}\right]
$$

The dissipation function $G$ can be expressed as:

$$
G=-\frac{1}{2} \int_{0}^{L} c\left(\frac{\partial \omega}{\partial t}\right)^{2} d x=-\frac{1}{2} \sum_{i} \sum_{j} \frac{\partial \phi_{i}}{\partial t} \frac{\partial \phi_{j}}{\partial t} \int_{0}^{L} c \theta_{i} \theta_{j} d x=-\frac{c}{2 m} \sum_{i} M_{i}\left(\frac{\partial \phi_{i}}{\partial t}\right)^{2}
$$

The virtual work done by the magnetic pressure $p(x, t)=q[1-H(x-v t)]$ in a virtual displacement $\delta \phi_{i}$ can be expressed as follows:

$$
\partial W=\int_{0}^{L} p(x, t) \delta w_{i} d x=\sum_{i} \partial \phi_{i} \int_{0}^{L} p(x, t) \theta_{i}(x) d x=\sum_{i} \partial \phi_{i} Q_{i}
$$

Where $Q_{i}$ is the generalized force

$$
\begin{aligned}
Q_{i} & =\int_{0}^{L} p(x, t) \theta_{i}(x) d x \\
& =\int_{0}^{v t} q \theta_{i}(x) d x \\
& =\frac{q}{\beta_{i}}\left[\left(\sinh \beta_{i} v t-\sin \beta_{i} v t\right)-\frac{\cosh \beta_{i} L+\cos \beta_{i} L}{\sinh \beta_{i} L+\sin \beta_{i} L} \times\left(\cosh \beta_{i} v t+\cos \beta_{i} v t-2\right)\right]
\end{aligned}
$$

Substituting $T, U, G, Q_{i}$ into the Lagrange equation which considering the damping force, we obtain an ordinary differential equation:

$$
\frac{\partial^{2} \phi_{i}}{\partial t^{2}}+\frac{c}{m} \frac{\partial \phi}{\partial t}+c \lambda_{i}^{2} \phi_{i}=\frac{Q_{i}(t)}{M_{i}}=\frac{q}{M_{i} \beta_{i}} F(t)
$$

Where:

$$
F(t)=\left(\sinh \beta_{i} v t-\sin \beta_{i} v t\right)-\frac{\cosh \beta_{i} L+\cos \beta_{i} L}{\sinh \beta_{i} L+\sin \beta_{i} L} \times\left(\cosh \beta_{i} v t+\cos \beta_{i} v t-2\right)
$$

The general solution of equation (24) is: 


$$
\begin{aligned}
\phi_{i}(t)= & \frac{m}{R}\left(\frac{\partial \phi_{i}(0)}{\partial t}+\frac{c+R}{2 m} \phi_{i}(0)\right) e^{\frac{-c+R}{2 m} t}+\frac{m}{R}\left(-\frac{\partial \phi_{i}(0)}{\partial t}+\frac{-c+R}{2 m} \phi_{i}(0)\right) e^{\frac{-c-R}{2 m} t} \\
& +\frac{q m}{M_{i} \beta_{i} R} \int_{0}^{t} F(\xi)\left(e^{\frac{-c+R}{2 m}(t-\zeta)}-e^{\frac{-c-R}{2 m}(t-\zeta)}\right) d \xi
\end{aligned}
$$

So the general solution of (1) can be expressed as follows:

$$
\begin{aligned}
w(x, t)= & \sum_{i} \theta_{i}(x) \phi_{i}(t) \\
= & \sum_{i}\left[\left(\cosh \beta_{i} x-\cos \beta_{i} x\right)-\frac{\cosh \beta_{i} L+\cos \beta_{i} L}{\sinh \beta_{i} L+\sin \beta_{i} L}\left(\sinh \beta_{i} x-\sin \beta_{i} x\right)\right] \\
& \times\left\{\frac{m}{R}\left(\frac{\partial \phi_{i}(0)}{\partial t}+\frac{c+R}{2 m} \phi_{i}(0)\right) e^{\frac{-c+R}{2 m} t}+\frac{m}{R}\left(-\frac{\partial \phi_{i}(0)}{\partial t}+\frac{-c+R}{2 m} \phi_{i}(0)\right) e^{\frac{-c-R}{2 m} t}\right. \\
& \left.+\frac{q m}{M_{i} \beta_{i} R} \int_{0}^{t} F(\xi)\left(e^{\frac{-c+R}{2 m}(t-\zeta)}-e^{\frac{-c-R}{2 m}(t-\zeta)}\right) d \xi\right\}
\end{aligned}
$$

Assuming that the initial conditions are as:

$$
\left\{\begin{array}{c}
\phi(0)=0 \\
\left.\frac{\partial \phi(t)}{\partial t}\right|_{t=0}=0
\end{array}\right.
$$

Equation (23) becomes:

$$
\begin{aligned}
\phi_{i}(t)= & \frac{q m}{M_{i} \beta_{i} R} \int_{0}^{t}\left[\left(\sinh \beta_{i} v \xi-\sin \beta_{i} \nu \zeta\right)-\frac{\cosh \beta_{i} L+\cos \beta_{i} L}{\sinh \beta_{i} L+\sin \beta_{i} L}\right. \\
& \left.\times\left(\cosh \beta_{i} \nu \zeta+\cos \beta_{i} \nu \zeta-2\right)\right] \times\left(e^{\frac{-c+R}{2 m}(t-\zeta)}-e^{\frac{-c-R}{2 m}(t-\zeta)}\right) d \xi
\end{aligned}
$$

Take an integration of (26), we get:

$$
\phi_{i}(t)=\frac{q m}{M_{i} \beta_{i} R}\left\{(1)-(2)-\left(\frac{\cosh \beta_{i} L+\cos \beta_{i} L}{\sinh \beta_{i} L+\sin \beta_{i} L}((3)+(4)-(5))\right)\right\}
$$

Where:

$$
\begin{aligned}
(1)= & \int_{0}^{t} \sinh \beta_{i} v \zeta\left(e^{\frac{-c+R}{2 m}(t-\zeta)}-e^{\frac{-c-R}{2 m}(t-\zeta)}\right) d \xi \\
= & 2 m\left[-R^{3} e^{\left(\beta_{i} v t\right)^{2}}-4 R \beta_{i} v m c-4 R \beta_{i}^{2} v^{2} m^{2}+4 R \beta_{i}^{2} v^{2} m^{2} e^{\left(\beta_{i} v t\right)^{2}}-4 R \beta_{i} v m c e^{\left(\beta_{i} v\right)^{2}}+R^{3}\right. \\
& +c^{2} R e^{\left(\beta_{i} v\right)^{2}}-c^{2} R+e^{\beta_{i} v t} e^{\frac{-c+R}{2 m} t}\left(2 m \beta_{i} v R^{2}+4 R \beta_{i} v m c-2 m \beta_{i} v c^{2}-8 m^{3} \beta_{i}^{3} v^{3}\right) \\
& \left.+e^{\beta_{i} v t} e^{\frac{-c-R}{2 m} t}\left(-2 m \beta_{i} v R^{2}+4 R \beta_{i} v m c+2 m \beta_{i} v c^{2}+8 m^{3} \beta_{i}^{3} v^{3}\right)\right] /\left[e ^ { \beta _ { i } v t } \left(16 \beta_{i}^{4} v^{4} m^{4}\right.\right. \\
& \left.\left.-2 c^{2} R^{2}+c^{4}+R^{4}-8 c^{2} m^{2} \beta_{i}^{2} v^{2}-8 R^{2} m^{2} \beta_{i}^{2} v^{2}\right)\right] \\
(2) & =\int_{0}^{t} \sin \beta_{i} v \xi\left(e^{\frac{-c+R}{2 m}(t-\xi)}-e^{\frac{-c-R}{2 m}(t-\xi)}\right) d \xi \\
& =4 m\left[4 \beta_{i} v m c R \cos \left(\beta_{i} v t\right)+R \sin \left(\beta_{i} v t\right)+4 R \beta_{i}^{2} v^{2} m^{2} \sin \left(\beta_{i} v t\right)-c^{2} R \sin \left(\beta_{i} v t\right)\right. \\
& -e^{\frac{-c+R}{2 m} t}\left(m \beta_{i} v R^{2}+\beta_{i} v m c^{2}+2 m \beta_{i} v c R-4 m^{3} \beta_{i}^{3} v^{3}\right)+e^{\frac{-c-R}{2 m} t}\left(m \beta_{i} v R^{2}+\beta_{i} v m c^{2}\right. \\
& \left.\left.-2 m \beta_{i} v c R+4 m^{3} \beta_{i}^{3} v^{3}\right)\right] /\left(c^{4}-2 c^{2} R^{2}+8 c^{2} m^{2} \beta_{i}^{2} v^{2}+R^{4}+8 R^{2} m^{2} \beta_{i}^{2} v^{2}+16 m^{2} \beta_{i}^{2} v^{2}\right)
\end{aligned}
$$




$$
\begin{aligned}
(3)= & \int_{0}^{t} \cosh \beta_{i} v \zeta\left(e^{\frac{-c+R}{2 m}(t-\zeta)}-e^{\frac{-c-R}{2 m}(t-\zeta)}\right) d \xi \\
= & 2 m\left[R^{3} e^{\left(\beta_{i} v\right)^{2}}-4 R \beta_{i} v m c-4 R \beta_{i}^{2} v^{2} m^{2}-4 R \beta_{i}^{2} v^{2} m^{2} e^{\left(\beta_{i} v t\right)^{2}}+4 R \beta_{i} v m c e^{\left(\beta_{i} v\right)^{2}}+R^{3}\right. \\
& -c^{2} R e^{\left(\beta_{i} v\right)^{2}}+e^{\beta_{i} v t} e^{\frac{-c+R}{2 m} t}\left(c R^{2}-c^{2} R-4 R \beta_{i}^{2} v^{2} m^{2}-c^{3}+R^{3}+4 c m^{2} \beta_{i}^{2} v^{2}\right) \\
& \left.+e^{\beta_{i} v t} e^{\frac{-c-R}{2 m} t}\left(c R^{2}+c^{2} R+4 R \beta_{i}^{2} v^{2} m^{2}-c^{3}-R^{3}+4 c m^{2} \beta_{i}^{2} v^{2}\right)\right] /\left[e ^ { \beta _ { i } v t } \left(16 \beta_{i}^{4} v^{4} m^{4}\right.\right. \\
& \left.\left.-2 c^{2} R^{2}+c^{4}+R^{4}-8 c^{2} m^{2} \beta_{i}^{2} v^{2}-8 R^{2} m^{2} \beta_{i}^{2} v^{2}\right)\right] \\
(4)= & \int_{0}^{t} \cos \beta_{i} v \zeta\left(e^{\frac{-c+R}{2 m}(t-\zeta)}-e^{\frac{-c-R}{2 m}(t-\zeta)}\right) d \xi \\
= & -2 m\left[8 \beta_{i} v m c R \sin \left(\beta_{i} v t\right)+2 c^{2} R \sin \left(\beta_{i} v t\right)-2 R^{3} \cos \left(\beta_{i} v t\right)-4 R \beta_{i}^{2} v^{2} m^{2} \cos \left(\beta_{i} v t\right)\right. \\
& -e^{\frac{-c+R}{2 m} t}\left(c^{3}+c^{2} R-c R^{2}+4 m c \beta_{i}^{2} v^{2} R^{2}-R^{3}-4 R \beta_{i}^{2} v^{2} m^{2}\right)+e^{\frac{-c-R}{2 m} t}\left(-c^{3}+c^{2} R+c R^{2}\right. \\
& \left.\left.-4 m c \beta_{i}^{2} v^{2} R^{2}-R^{3}-4 R \beta_{i}^{2} v^{2} m^{2}\right)\right] /\left(c^{4}-2 c^{2} R^{2}+8 c^{2} m^{2} \beta_{i}^{2} v^{2}+R^{4}+8 R^{2} m^{2} \beta_{i}^{2} v^{2}+16 m^{2} \beta_{i}^{2} v^{2}\right) \\
& =\frac{4 m}{-c^{2}+R^{2}}\left[-2 R+(c+R) e^{\frac{-c+R}{2 m} t}+(c+R) e^{\frac{-c-R}{2 m} t}\right] \\
& (5)=2 \int_{0}^{t}\left(e^{\frac{-c+R}{2 m}(t-\zeta)}-e^{\frac{-c-R}{2 m}(t-\zeta)}\right) d \xi \\
&
\end{aligned}
$$

Substituting(31) - (35)into(30), then the substituting the calculating result into(27), can obtain the general solution $_{w(x, t)}$ of(1). The moment and the shear force of the beam in the rail can be further derived from $w(x, t)$, which provides a theoretical method for the overall investigation of the dynamic behavior of the electromagnetic railgun.

\section{Numerical example}

Since there are differences among materials of electromagnetic rail launcher, the damping force and the rate of moving load will possibly bring influence to the response of the rail. As the result, it is necessary to consider the damping force and the rate of moving load and to compare the response of the rail.

Sitting the governing equation of beam by using the below parameter(Anthony J. Johnson \& Francis C.Moon. 2005); modulus of rail material $E=120 \mathrm{GPa}$, the moment of inertia of the rail cross section $I=2.5 \times 10^{-9} \mathrm{~m}^{4}$, the elastic constant $k=2.532 \times 10^{10} \mathrm{~N} / \mathrm{m}^{2}$, the density of rail material $\rho=8320 \mathrm{~kg} / \mathrm{m}^{3}$, the width of rail $B=3 \times 10^{-2} \mathrm{~m}$, the thickness of rail $h=1 \times 10^{-2} \mathrm{~m}$, the magnetic pressure $q=100 \mathrm{MPa}$, the length of rail $L=2 \mathrm{~m}$, the velocity of projectile $v=1000 \mathrm{~m} / \mathrm{s}$, Accordingly, the time of armature's movement in the rail is $t=2 \times 10^{-3}$.

Figure. 3 shows the dynamic response of the elastic beam by the rate of moving load. It confers that the deformation $(w)$ is larger when the rate of moving load $(v)$ is bigger at the $0-1.6 \times 10^{-3} \mathrm{~s}$.

Figure. 4 shows the dynamic response of the beam by the damping coefficient. Along with the damping coefficient $(c)$ increasing, the curve of time-deformation is a decreasing trend. Under the calculating conditions given by this paper, for the rail of which $c$ equals to $1.3 \times 10^{5} \mathrm{Ns} / \mathrm{m}$, the deformation $(w)$ of the beam is $2.95 \times 10^{-3} \mathrm{~m}$ when the armature moves to the moment $t=1.2 \times 10^{-3}$. While for the rail of which $c$ equals to $6.5 \times 10^{5} \mathrm{Ns} / \mathrm{m}$, the deformation $(w)$ of the beam is $2.6 \times 10^{-3} \mathrm{~m}$ at the same moment, we see that the latter is $8.5 \%$ smaller than the former.

\section{Conclusions}

(1) Regarding the rail as a cantilever beam on the elastic foundation and considering the damping force, building a mathematic model for the electromagnetic railgun.

(2) Making use of variable method and the Lagrange equation which considering the damping force, The general solution of the homogeneous part and the complete solution of the governing equation is derived

(3) The dynamic response of the beam which is influenced by the velocity of moving load and damping coefficient are analyzed by the MATLAB software. With the increasing time, the response of beam is more obvious when the rate of the moving load is more larger. While the deformation is much smaller when the damping coefficient becomes larger. 


\section{References}

Anthony J. Johnson \& Francis C.Moon. (2005). Elastic Waves and Solid Armature Contact Pressure in Electromagnetic Launchers Transactions on Magnetics. 3,472-475.

$\mathrm{Hu}$, Yuwei. (2007). Modeling and simulation of electromagnetic rail gun system: [Dissertation for the Master Degree]. Harbin Institute of Technology.

Jerome T. Tzeng \&Wei, Sun. (2007). Dynamic Response of Cantilevered Rail Guns Attributed to Projectile/Gun Interaction -Theory. Transactions on Magnetics, 43: 207-213

Jerome T. Tzeng (2005), Dynamic Response of Electromagntic Railgun Due to Projectile Movement Transactions on Magnetics, 41: 246-250.

Jerome T. Tzeng. (2005). Structural Mechanics for Electromagnetic Railguns Transactions on Magnetics,1, $246-250$.

Jin, Shangnian, Ma, Yongli. (2002). Theoretical Mechanics. Higher Education Press, (chapter 7)

Liu, Xiaoyun, Tian, Runli. (2007). Dynamic Response Solution in Transient State of Viscous-elastic Road under Moving Load. Chinese Journal of Engineering Mathematics, 12:1049-1054.

Lou, Ping \& Zeng, Qingyuan. (2003). Finite element analysis of infinitely long beam resting on continuous viscoelastic foundation subjected to moving loads. Journal of Traffic and Tran sportation Engineering 3: 1-6.

S.Timoshenko. (1965). Mechanics of material. Science press (chapter 1)

Wang, Ying\& Xiao, Feng. (1994). Principle of electricgun. National Defense Industry Press, (chapter 1)

Weng, Xuetao \& Hu'. (2008). An. Response of an elastically supported infinite Timoshenko beam to moving vibrating mass. Journal of Naval University of Engineering, 20:65-68.

Yu, Yanli. (2002). The research of the dynamic response of the rail system and viaduct by moving load:[Dissertation for the Master Degree]. Wuhan University of Technology, 2002.

Zhang, Xiangting, Wang, Zhipei. (2006). Structure Vibration Mechanics. TongJi Univesity Press, (chapter 4)

Zhu, Shijian, Lou, lingjun. (2006). Vibration Theory and Vibration Isolation. National Defense Industry Press (chapter 2)

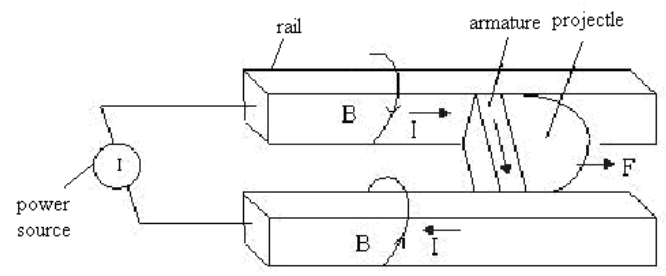

Figure 1. The general diagram of the railgun

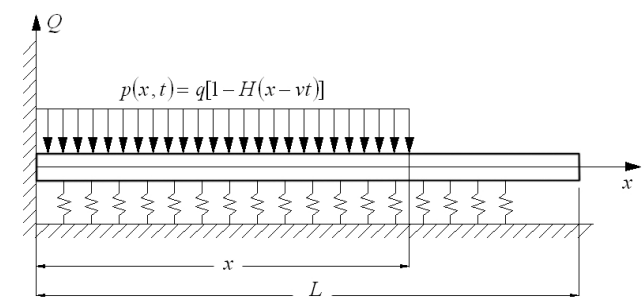

Figure 2. The rail is modeled as a beam on elastic foundation 


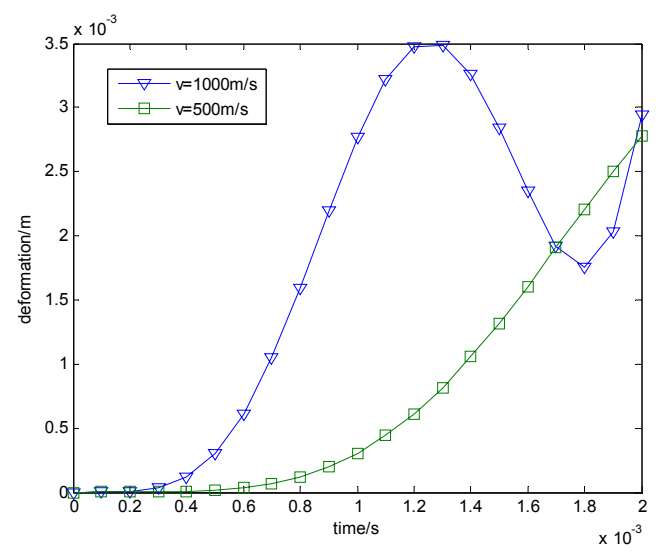

Figure 3. Deformation curve of elastic foundation beam by moving load of differences velocity

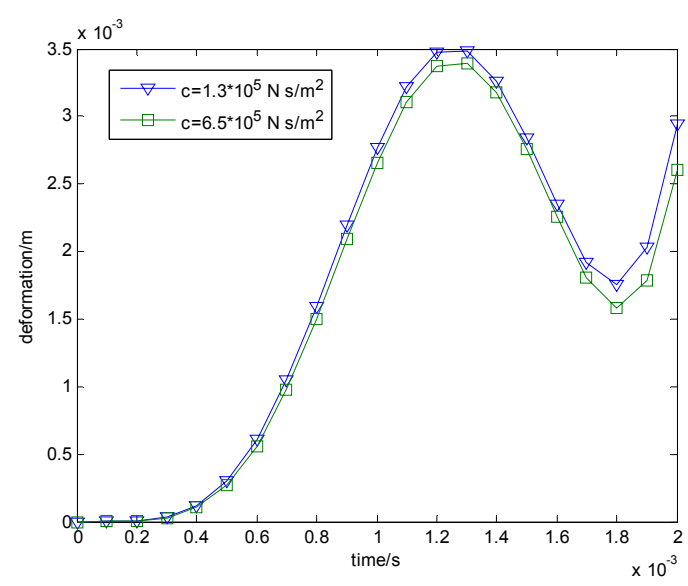

Figure 4. Deformation curve of by differences damping coefficient 